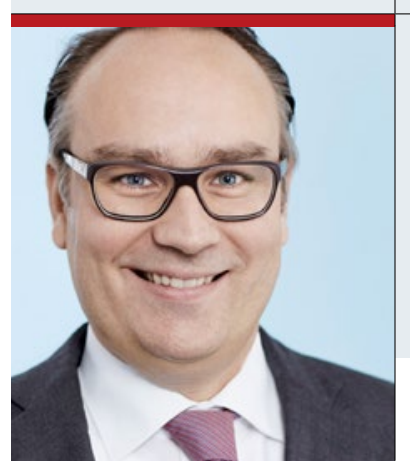

Falk H. Miekley

Director Professional Care

falk.miekley@springer.com

facebook.com/springerpflege

\title{
Kongress Pflege 2018: Mega-Thema Personal
}

$\mathrm{N}$ atürlich war klar, dass der neue Pflegebegriff und die neue Form der Begutachtung mehr Empfänger von Leistungen nach sich ziehen. Doch wie hoch die Zahl ist, hat uns dann doch überrascht: In den ersten zehn Monaten des Jahres 2017 sind 660.000 neue Leistungsempfänger nach dem neuen Verfahren anerkannt worden. 660.000! Das schreibt in dieser Ausgabe ein Mann, der es wissen muss: Dr. Peter Pick, Chef des Medizinischen Dienstes des Spitzenverbandes der Krankenkassen (MDS). Erwartungsgemäß „profitieren" vor allem Menschen mit Demenz und anderen gerontopsychiatrischen Beeinträchtigungen vom neuen System. Mit der Folge, dass insbesondere die ambulanten Pflegedienste gefragt sind, die das Personal für die Betreuungs- und Entlastungsangebote bereithalten müssen. Doch wie die Pflege-Wirklichkeit aussieht, wissen Sie selbst am allerbesten: Weder die ambulanten Dienste noch die Pflegeheime verfügen über genügend Personal, um diese Mehrarbeit leisten zu können.
Auch aus diesem Grund drehen sich bei unserem Kongress Pflege 2018 viele Veranstaltungen um das Mega-Thema Personal: Was genau bedeuten Personaluntergrenzen, wie wirken sie sich auf die Praxis aus? Wie gewinne ich Mitarbeiter und halte sie? Wie fördere ich talentierte Nachwuchskräfte?

Seien Sie herzlich eingeladen, diese und andere Themen mit uns zu diskutieren. Auch feiern möchten wir mit Ihnen: bei der Verleihung des Pflegemanagement-Awards im Rahmen des Kongresses. Wir von Springer Pflege freuen uns auf einen gemeinsamen Start in das Fortbildungsjahr 2018!

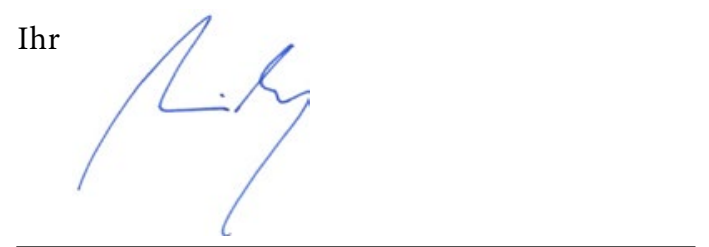

Anregungen, Wünsche, Fragen oder Lob? Schreiben Sie uns: falk.miekley@springer.com

\section{DIE GEWINNER STEHEN FEST}

Die Pflege und Betreuung von Menschen mit Demenz ist Königin Silvia von Schweden ein sehr persönliches Anliegen. Um angehende Pflegekräfte zu inspirieren, sich aktiv in die zukünftige Entwicklung der Pflege einzubringen, hat sie mit dem Queen Silvia Nursing Award ein Stipendium für Nachwuchskräfte in der Pflege gestiftet. Die Auszeichnung, die schon in mehreren europäischen Ländern vergeben

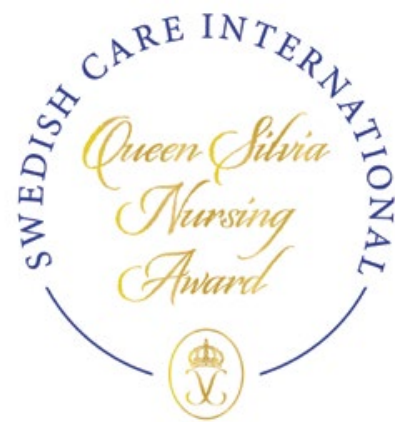

wird, wird jetzt erstmals auch an eine deutsche Pflegekraft verliehen. Diese reist gemeinsam mit den Preisträgern aus Schweden, Finnland und Polen nach Stockholm, wo alle von Königin Silvia im Schloss ausgezeichnet werden.

Zuvor können Sie die Preisträger am 19. Januar auf dem Kongress Pflege 2018 in Berlin erleben.

www.queensilvianursingaward.de 